
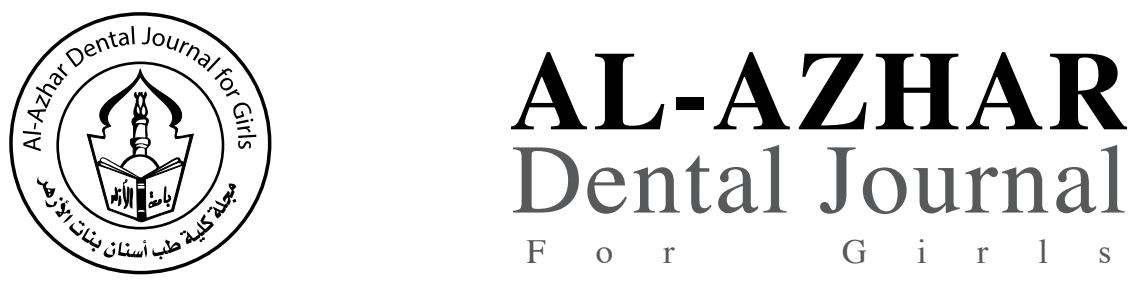

The Official Publication of The Faculty of Dental Medicine For Girls,

Al-Azhar University F o r

G i

Cairo, Egypt.

ADJ-for Grils, Vol. 3, No. 1, January (2016) - PP. 55:60

\title{
Effect of Different Doses of Power Horse Energy Drink on Submandibular Salivary Glands of Albino Rats
}

\author{
Abeer A.A. Mohamed ${ }^{(1)}$, Mona H.M. Farid ${ }^{(2)}$, Heba A. Adawy ${ }^{(2)}$ and Marwa A. El-Shiekh ${ }^{(2)}$
}

Codex : 08/1601

dentaljournal.forgirls@yahoo.com

\begin{abstract}
Objective: The aim of this study is to investigate histological the effect of power horse energy drink intake with different doses on the submandibular salivary glands of albino rats. The possible modulated recovery of expected adverse effects from the different doses of the taken power horse energy drink. Materials and Methods: Sixty five adult male albino rats (weighing 180-200 grams) were divided equally into seven groups. Group I (control), group (IIA,IIIA\&IVA) which received a daily different dose power horse energy drink using an oro-pharyngeal metallic curved tube for 8 weeks then sacrified and group(IIB,IIIB\&IVB)which left alive with stoppage of beverage intake for another 4 weeks for recovery then scarification were done. The specimen were stained by H\&E. Result: Power horse energy drink intake result in adverse effects on the submandibular salivary glands in the form of structural changes of all gland elements including: These changes are dose dependant and showed in the following:Atrophy of secretory units, loss of normal glandular architecture, numerous signs of degeneration and premalignant changes. Signs of degeneration of striated.
\end{abstract}

\section{INTRODUCTION}

Energy drinks are non-alcoholic, lightly carbonated beverages that are designed to give the consumer a dose of energy. They have become very popular all over the world since 1997 . Nowadays, they are more popular than ever and their popularity seems to increase every year, they are used frequently with teenagers, young adults, athletes and physically active subpopulations. Energy drinks are typically attractive to young people. Adolescents and young adults are often uninformed about the content of energy drink. ${ }^{(1,2)}$ Approximately 65\% percent of its drinkers are between the ages of 13 and 35 years old, with males

1. Demonstrator of Oral and Dental biology Department, Faculty of Dental Medicine, MTI University.

2. Oral and Dental biology Department, Faculty of Dental Medicine for Girls, AL-Azhar University. 
representing approximately $65 \%$ of the consumers. (3) These drinks are marketed as natural alternatives that increase fun and improve physical and cognitive performance such as concentration, attention and alertness. $^{(4)}$ Histopathological observations of kidney tissue in energy drinks administered animals, revealed distinct pathological lesions as represented by necrosis of renal tubules and glomeruli as well as intertubular hemorrhage and leucocytic infilteration. Moreover, electron microscopic results showed marked ultrastructure alterations in the nucleous and cytoplasmic oraganelles in the cells of proximal and distal tubules as well as in the renal corpuscles. These alterations were more obvious in rats given power horse. ${ }^{(5)}$ Administration of Power Horse like beverage (with caffeine and taurine) significantly increased the contractility of the left atrium that accounts for the higher left end-diastolic volume leading to an increase in stroke volume. ${ }^{(6)}$ Power horse was more effective in its action on liver enzymes, followed by red bull and to less extend code red. The different action of the energy drinks on liver function could be attributed to the different mixture of their ingredients. ${ }^{(7)}$

\section{MATERIALS AND METHODS}

Sixty five healthy adult male albino rats weighing 180-200 grams were used in this study. The experiment were done in the animal house of Plant Protection Research Institute. The rats were maintained under normal laboratory conditions and kept in standard cages at room temperature of $25^{\circ} \mathrm{c}$ $30^{\circ} \mathrm{C}$ and provided with standard diet and water $a d-$ libitum.The animals were divided into seven groups as the follows:

A control group (group I): consisted of five rats with phosphate buffering.

Experimental groups: consisted of 3 groups: Group II: consisted of 20 rats which received a daily single dose $(3.57 \mathrm{ml} / \mathrm{kg} \mathrm{b} \text {. wt. })^{(39)}$ of power horse

Solution using an oropharyngeal metallic curved tube for 8 weeks. This dose is equivalent to the minimal human dose (1can $(330 \mathrm{ml})$ /day) in relation to the animal's body weight. Then the group was equally divided into two subgroups (A\&B) (10 rats for each group) as follows: Group II (A) was sacrificed after 8 weeks from the beginning of the experiment. Group II (B) was left alive with stoppage of beverage intake for another 4 weeks for recovery then were sacrificed (meaning that the scarification were done after 12 weeks from the beginning of the experiment).Group III: consisted of 20 rats which received a daily single dose ( $10.71 \mathrm{mlkg}$ b.wt.) of power horse for 8 weeks which is equivalent to human dose (3cans /day) in relation to the animal's body weight and then, the group was equally divided into two subgroups (A\&B) (10 rats for each group) as follows: Group III (A) was sacrificed after 8 weeks from the beginning of the experiment. Group III (B) was left alive with stoppage of beverage intake for another 4 weeks for recovery then were sacrificed (meaning that the scarification were done after 12 weeks from the beginning of the experiment).Group IV: consisted of 20 rats which received a daily single dose $(17.86 \mathrm{ml} / \mathrm{kg} \mathrm{b}$. wt.) of power horse for 8 weeks that is equivalent to human dose (5cans /day) and in relation to the animal's body weight. This group was then equally divided into two subgroups (A\&B) (10 rats for each group) as follows: Group IV (A) was sacrificed after 8 weeks from the beginning of the experiment. Group IV (B) was left alive with stoppage of beverage intake for another 4 weeks for recovery then they were sacrificed (meaning that the scarification were done after 12 weeks from the beginning of the experiment).

The scarification of rats were done by cervical decapitation. The submandibular salivary glands were dissected free and cleaned rapidly of any adherent connective tissue. The specimens of the submandibular salivary glands were prepared for routine histological examination using haematoxylin and eosin stain 


\section{RESULTS}

\section{Group I (Control group):}

Microscopic examination of the rat submandibular glands of control group showed mixed acini, striated ducts and granular convoluted tubules in between. The mixed acini were lined by secretory cells having uniform rounded basophilic nuclei. The granular convoluted tubules were lined by columnar cells having central rounded nuclei and characterized by presence of numerous eosinophilic granules. The striated ducts were lined by columnar cells having oval nuclei (Fig.1A)

\section{GroupIIA (administerated low dose of power horse):}

Histological examination of the submandibular glands of this group obtained a low dose of energy drink revealed shrunken, widely spaced acini. Destructive changes were observed revealed by degeneration of some acini leaving empty spaces and the remaining acini lacked the normal architecture. Some serous acini showed hyalinization of the lining cells with loss of normal architecture of the acini. The lining epithelial cells of the acini showed variation in size and shape with deep basophilic nuclei. Some of them showed numerous mitotic figures. Other nuclei showed vaculization and vacuolar degenerated cytoplasm while the interstitial stoma between the acini and lobules was homogenous deeply eosinophilic with lose of fibrillar appearance (Fig.1B)

\section{Group IIIA (administrated medium dose of power horse):}

In the specimens of this group, there were marked loss of normal acinar architecture and normal cell arrangment (amalgamation) leaving variable sized edematous spaces in between serous acini. This was beside the presence of extensive vacuolated cytoplasm, the nuclei exhibited different sizes and shape (pleomorphism) as well as increased basophilia (hyperchromatism).
Numerous mitotic figures were also demonstrated. On examining striated ducts, they showed mild degree of degeneration demonstrated with loss of normal architecture in the form of intracellular vacuolization, poorly distinct boundaries were also detected between the lining cells of the duct with loss of normal lining cell arrangement (Fig.1C).

Group IV A (Group or rats administrated high dose): The submandibular glands taking high dose of energy drink revealed shrunken, widely spaced serous acini with many cytoplasmic vacuoles of their cell lining. The gland tissue appeared less compact with the appearance of some spaces and clefts between the acini. There was an obvious degenerated acini with marked loss of normal structure and architecture. The cells lining of the acini are hardly detected and differentiated from each other. They appeared as haphazardly arranged nuclei with no distinct cell boundaries. The nuclei became more deeply stained (Hyperchromatism) with abnormal mitotic figures and various degree of polymorphism. Striated ducts also showed clear changes where the lining cells were highly distorted, the cell boundaries were not seen, the lumen is stenosed and appeared narrow or even missing and without definite borders (Fig.1D).

\section{Recovery groups:}

Group of recovered rats of low dose (IIB): On examining the specimens of this group, the gland tissue showed some recovery changes where, the acini revealed some improvement in their architecture and structure. The lining cells became more normally arranged in a proper alignment. The nuclei of the lining cells appeared normal without any vesicular appearance, while this vesicular appearance persist in the cytoplasm of most of the cells. The striated ducts revealed the most clear evidence of regeneration as they appeared normal in size, shape and lumen size but with cytoplasmic vacuolization. In few areas of the examined specimen, some degenerative features were still present in the form of degenerated acini (Fig.2A). 
Group of recovered rats of medium dose (IIIB): The acini of this group did not show much regeneration, most of the degenerative changes were observed; although there were minimal amount of vesicular nuclei of the lining cells, but still there is a lot of nuclear pleomorphism, some mitotic figures and also nuclear fragmentation were also seen. Some areas of the examined gland showed restoration for the architecture of the acini while others showed amalgamation of the nuclei without any normal alignment. Furthermore, some of the acini showed hyalinization and loss of cell boundaries with the presence of remnants of nuclei and sometimes complete disappearance of nuclei. Some striated duct showed normal lining but with loss of cell boundaries and narrow lumen, others showed incomplete lining of the duct, while others failed to regenerate where complete hyalinization and loss of structure were seen with complete absence of the duct lumen (Fig.2B).

\section{Group of recovered rats of high dose (IVB):} Serous acini showed some degree of regeneration where the nuclei appeared with normal size, shape and stainability but still some signs of degenerative

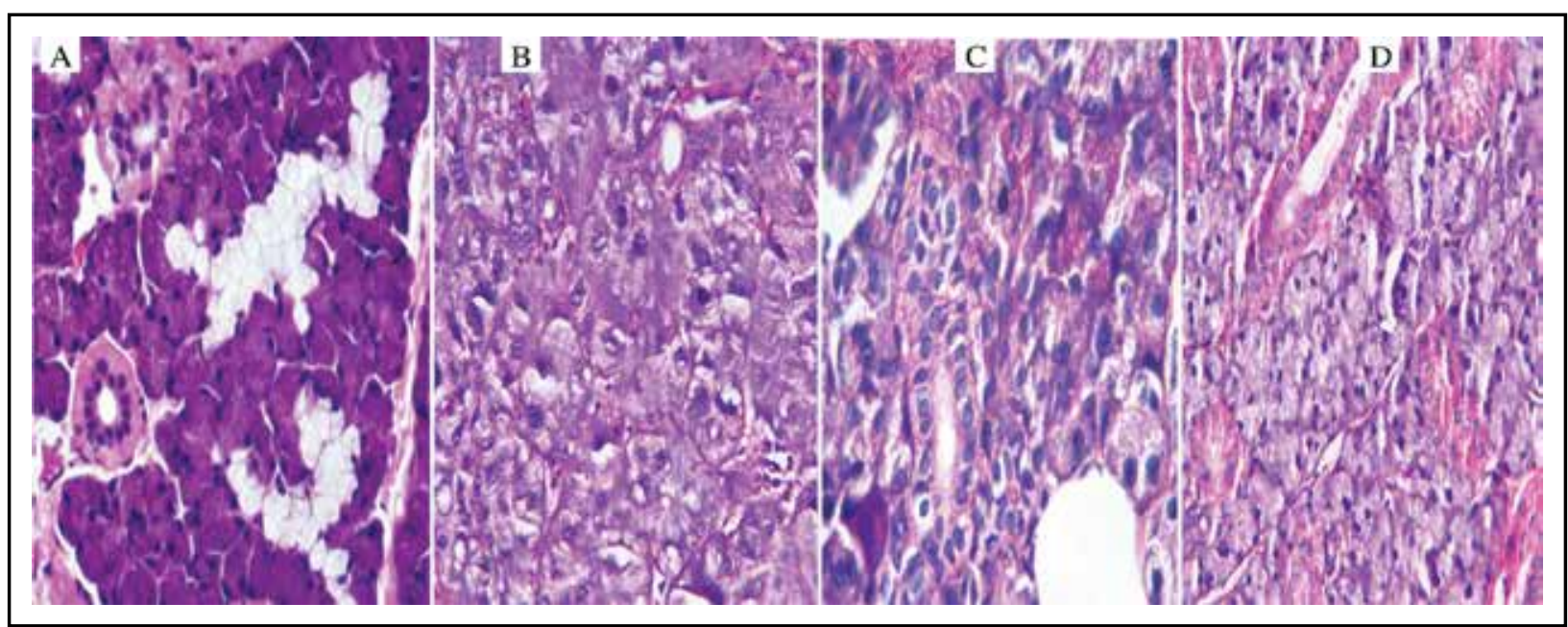

Fig. (1) A Photomicrograph of submandibular salivary gland showing GI with normal architecture (A), GII, III \& IV showing variable degeneration of normal architecture (B,C\&D) respectively. (H \& E Orig. Mag. X 200)

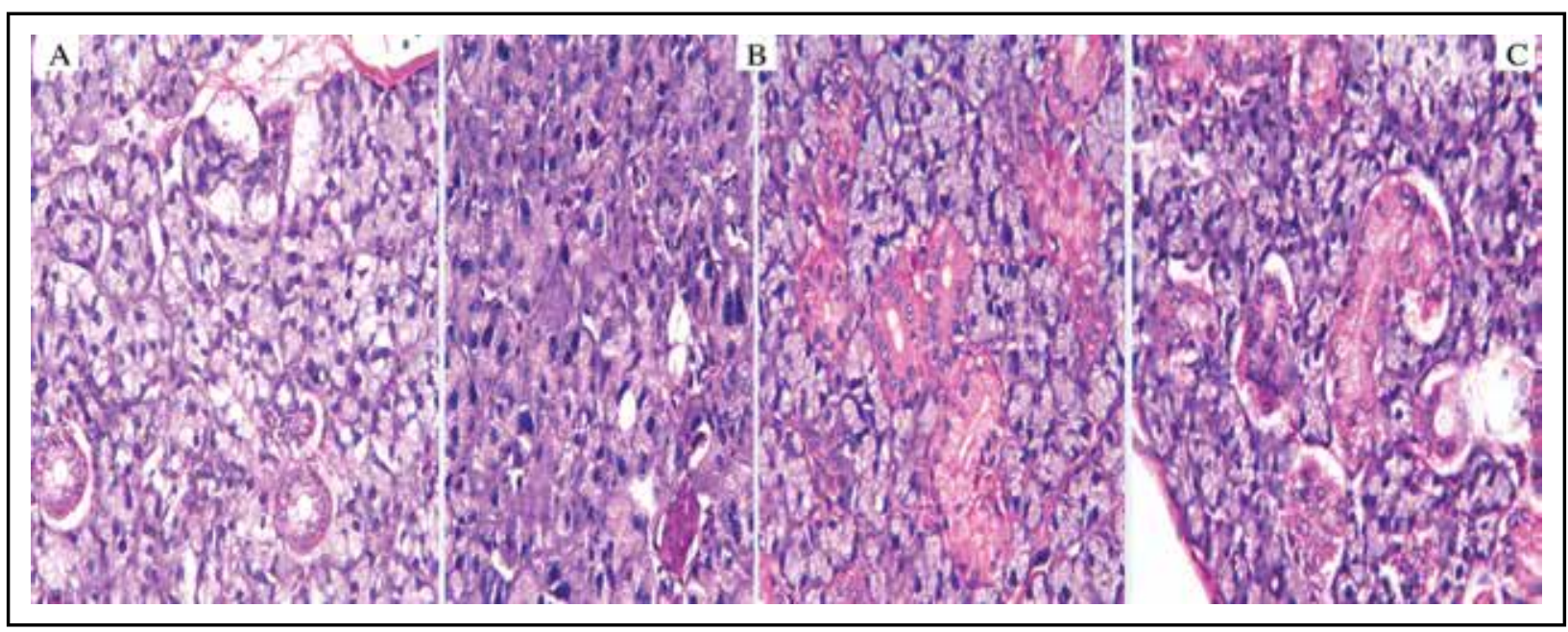

Fig. (2) A photomicrograph of SMG of recovery group in GIIB showing improvement in structure and architecture. While recovered group of medium dose (GIIIB) showed medium regeneration as there were minimal amount of vesicular nuclei and high dose (GIVB) showed minimal amount of recovery. (H \& E Orig. Mag. X 200) 
influence of the energy drink seen in the form of loss of the proper, normal architecture of the acini. The striated ducts also did not reveal much regenerative signs as they showed obliterated lumens, habhazared arrangement of the lining cells with vesicular nuclei and sometimes completely degenerated nuclei. Moreover, some of the striated ducts showed marked atrophy and sometimes completely disappeared (Fig.2C).

\section{DISCUSSION}

Light microscopic examination revealed numerous intracellular vacuolization appeared mainly in serous acini and striated ducts of all groups receiving variable doses of energy drink. This finding might be attributed to degenerative changes within the secretory portions that were mostly of fatty nature (fatty degeneration). However, in the routinely processed hematoxylin and eosin sections the lipid droplets were dissolved during fixation and processing of the tissues leaving empty vacuoles. This was consistent with Mubarak (2012), who studied the effect of Red Bull energy drink on Rats' Submandibular salivary glands..$^{(8)}$

Histological examination of the submandibular glands of (GIIA, IIIA \& IVA) obtained different dose of energy drink revealed atrophy or shrunken, widely spaced acini. These fingings might be due to high amount of sugar present in energy drink. These were agreed with Bukhar (2012) who found that high energy drink cause histopathological changes in the liver for normal rat and diabetic rat like atrophy and cell damage also changes in the chemical and morphological structure. ${ }^{(9)}$

On elevated doses of energy drinks the nuclei of the secretory cells showed nuclear pleomorphism and hyperchromatism. Numerous mitotic figures were also detected. All these findings were considered as signs of premalignancy. These premalignat changes might be due to the preservatives added to the energy drinks as sodium Benzoate. Sodium benzoate is a type of salt that may occur naturally in some foods but is chemically produced and added as a preservative to energy drinks. There were health concerns about the combination of sodium benzoate and ascorbic acid, another common ingredient in energy drinks. When these two substances were mixed, they could form the chemical benzene, which is carcinogenic. (10) $^{-}$

In the current study, the recovered group of low dose showed improvement in architecture and structure of their acini. The lining cells became more normaly arranged in a proper alignment. The nuclei of some lining cells appeared normal without any vesicular appearance. These findings were in agreement of Akande and Banjoko (2011) (11), where they found that the histopathological study of the hepatocytes of rats in the recovery group showed moderate architectural distortion where the hepatocytes showed mixed morphology i.e. some cells were regenerating, thereby there was a gradual loss of the vacuole and reappearance of the cytoplasm around the cells were observed. Moreover, they recommended further research on the effects of excessive consumption over a longer period of time, with a longer recovery phase to determine if a total recovery is possible on cessation.

While recovered group of medium dose (GIIIB) showed medium regeneration as there were minimal amount of vesicular nuclei and high dose (GIVB) showed minimal amount of recovery which appeared more degenerated as the condition become irreversible. These findings were in disharmony with the results of Akande and Banjoko $(2011)^{(11)}$, where they stated that the damage done by excessive consumption of caffeinated energy drink is reversible as observed in the results of the blood chemistry analysis and the histopathological study of the organs of animals in the recovery group. On the other hand These findings were in harmony with Khayyat et al. (2014) ${ }^{(5)}$ where they suggested that energy drinks may cause irreversible structural changes in rat renal tissue, which could play an important role in renal dysfunction. 


\section{REFERENCES}

1. Rath M.: Energy drinks: What is all the hype? The dangers of energy drink consumption. Journal Of The American Academy Of Nurse Practitioners. 2012 ; 24, No. 2, 70-76.

2. Chad J, Eric C, Roland R: Caffeinated energy drinks- A Growing Problem. Drug Alcohol Depend. 2009; 99:1-10.

3. Mintel Energy Drink Report 2006, 07.05.06.

4. Kaminer Y: Problematic use of energy drinks by adolescents. Child Adolesc. Psychiatr. Clin. Am. 2010; 19:643-650.

5. Khayyat L., Essawy A., Sorour J., and Al Rawi M.: Impact of Some Energy Drink on the Structure and Function of the Kidney in Wistar Albino Rats. Life Sci. J.2014; 11(10):1131-1138.

6. Baum M, Weiss M: The influence of taurine containing drink on cardiac parameters before and after exercise measured by echocardiography. Amino Acids. 2001; 20:75-82.
7. Khayyat L, Sorour J, Al Rawi M, Essawy A: Histological,ultrastracture and physiological studies on the effect of different kinds of energy drinks on the liver of wister albino rat. J. Am. Sci. 2012;8:688-697.

8. Mubarak R: Effect of red bull energy drink on rat's submandibular salivary glands (light and electron microscopic study). Journal of American Science 2012; 8:366-372.

9. Bukhar H.M., ElSawy N.A., Header E.A. Biological Effect of High Energy Drink on Normal and Hyperglycemic Rats Pakistan Journal of Nutrition 2012;11 (4): 301-309.

10. Plant M. Should You Be Drinking Energy Drinks? November 17th, 2008. http://plantmiracle.com.

11. Akande I. S. and Banjoko O.A.: Assessment of Biochemical Effect of "Power Horse" Energy Drink on Hepatic, Renal and Histological Functions in Sprague Dawley Rats. Annual Review \& Research in Biology, 2011; 1(3): 45-56. 